\title{
ON IDENTITIES OF INFINITE DIMENSIONAL LIE SUPERALGEBRAS
}

\author{
DUŠAN REPOVŠ AND MIKHAIL ZAICEV
}

(Communicated by Kailash C. Misra)

\begin{abstract}
We study codimension growth of infinite dimensional Lie superalgebras over an algebraically closed field of characteristic zero. We prove that if a Lie superalgebra $L$ is a Grassmann envelope of a finite dimensional simple Lie algebra, then the PI-exponent of $L$ exists and is a positive integer.
\end{abstract}

\section{INTRODUCTION}

We shall consider algebras over a field $F$ of characteristic zero. One of the approaches in the investigation of associative and non-associative algebras is to study numerical invariants associated with their identical relations. Given an algebra $A$, we can associate to it the sequence of its codimensions $\left\{c_{n}(A)\right\}_{n \in \mathbb{N}}$ (all notions and definitions will be given in the next section).

This sequence gives some information not only about the identities of $A$ but also about the structure of $A$. For example, $A$ is nilpotent if and only if $c_{n}(A)=0$ for all large enough $n$. If $A$ is an associative non-nilpotent $F$-algebra, then $A$ is commutative if and only if $c_{n}(A)=1$ for all $n \geq 1$.

For an associative algebra $A$ with a non-trivial polynomial identity, the sequence $c_{n}(A)$ is exponentially bounded by the celebrated Regev Theorem [20], while $c_{n}(A)=n$ ! if $A$ does not satisfy any non-trivial polynomial identity. In the non-associative case the sequence of codimensions may have even faster growth. For example, if $A$ is an absolutely free algebra, then

$$
c_{n}(A)=a_{n} n !
$$

where

$$
a_{n}=\frac{1}{2}\left(\begin{array}{c}
2 n-2 \\
n-1
\end{array}\right)
$$

is the Catalan number, i.e. the number of all possible arrangements of brackets in the word of length $n$.

Received by the editors April 17, 2011 and, in revised form, December 9, 2011; January 4, 2012; and February 8, 2012.

2010 Mathematics Subject Classification. Primary 17C05, 16P90; Secondary 16R10.

Key words and phrases. Polynomial identity, Lie algebra, codimension, exponential growth.

The first author was supported by the Slovenian Research Agency grants P1-0292-0101 and J1-4144-0101.

The second author was partially supported by RFBR grant No. 13-01-00234a.

Both authors thank the referee for several comments and suggestions. 
For a Lie algebra $L$ the sequence $\left\{c_{n}(L)\right\}_{n \in \mathbb{N}}$ is in general not exponentially bounded, even if $L$ satisfies non-trivial Lie identities (see for example [18]). Nevertheless, a class of Lie algebras with exponentially bounded codimensions is sufficiently wide. It includes, in particular, all finite dimensional algebras [1,11, KacMoody algebras [23, 24], infinite dimensional simple Lie algebras of Cartan type [15], Virasoro algebra, and many others.

In the case when $\left\{c_{n}(A)\right\}_{n \in \mathbb{N}}$ is exponentially bounded, the upper and lower limits of the sequence $\left\{\sqrt[n]{c_{n}(A)}\right\}_{n \in \mathbb{N}}$ exist and a natural question arises: does the ordinary limit

$$
\lim _{n \rightarrow \infty} \sqrt[n]{c_{n}(A)}
$$

exist? In the case of existence we call this limit $\exp (A)$ or the PI-exponent of $A$.

Amitsur conjectured in the 1980's that for any associative P.I. algebra such a limit exists and that it is a non-negative integer. This conjecture was confirmed first for verbally prime P.I. algebras in [4,21] and later for the general case in [8,9]. For Lie algebras a series of positive results was obtained for finite dimensional algebras [6, 7, 25, for algebras with nilpotent commutator subalgebras [17, for affine KacMoody algebras [23,24], and for some other classes (see [16]). For Lie superalgebras there exist only partial results $[26,27,30,31]$.

On the other hand, it was shown in [28] that there exists a Lie algebra $L$ with

$$
3.1<\liminf _{n \rightarrow \infty} \sqrt[n]{c_{n}(L)} \leq \limsup _{n \rightarrow \infty} \sqrt[n]{c_{n}(L)}<3.9
$$

This algebra $L$ is soluble and almost nilpotent; i.e. it contains a nilpotent ideal of finite codimension. In the general non-associative case there exists, for any real number $\alpha>1$, an algebra $A_{\alpha}$ such that

$$
\lim _{n \rightarrow \infty} \sqrt[n]{c_{n}\left(A_{\alpha}\right)}=\alpha
$$

(see [5]). Note also that by a recent result [12] there exist finite dimensional Lie superalgebras with a fractional limit $\sqrt[n]{c_{n}(L)}$.

In the present paper we shall study Grassmann envelopes of finite dimensional simple Lie algebras. Our main result is the following theorem:

Theorem 1. Let $L_{0} \oplus L_{1}$ be a finite dimensional simple Lie algebra over an algebraically closed field $F$ of characteristic zero with some $\mathbb{Z}_{2}$-grading. Also, let $\widetilde{L}=L_{0} \otimes G_{0} \oplus L_{1} \otimes G_{1}$ be the Grassmann envelope of $L$. Then the limit

$$
\exp (\widetilde{L})=\lim _{n \rightarrow \infty} \sqrt[n]{c_{n}(\widetilde{L})}
$$

exists and is a positive integer. Moreover, $\exp (\widetilde{L})=\operatorname{dim} L$.

Another result of our paper concerns graded identities. Since any Lie superalgebra $L$ is $\mathbb{Z}_{2}$-graded, one can consider $\mathbb{Z}_{2}$-graded identities of $L$ and the corresponding graded codimensions $c_{n}^{g r}(L)$. We shall prove that graded codimensions have similar properties. 
Theorem 2. Let $L=L_{0} \oplus L_{1}$ be a finite dimensional simple Lie algebra over an algebraically closed field $F$ of characteristic zero with some $\mathbb{Z}_{2}$-grading. Also, let $\widetilde{L}=L_{0} \otimes G_{0} \oplus L_{1} \otimes G_{1}$ be a Grassmann envelope of $L$. Then the limit

$$
\exp ^{g r}(\widetilde{L})=\lim _{n \rightarrow \infty} \sqrt[n]{c_{n}^{g r}(\widetilde{L})}
$$

exists and is a non-negative integer. Moreover, $\exp ^{g r}(\widetilde{L})=\operatorname{dim} L$.

In other words, both PI-exponent $\exp (\widetilde{L})$ and graded PI-exponent $\exp ^{g r}(\widetilde{L})$ exist, they are integers, and they coincide. Note that for an arbitrary $\mathbb{Z}_{2}$-graded algebra the growth of ordinary codimensions and graded codimensions may differ. For example, if $A=M_{k}(F) \otimes F \mathbb{Z}_{2}$ with the canonical $\mathbb{Z}_{2}$-grading induced from group algebra $F \mathbb{Z}_{2}$, where $M_{k}(F)$ is a full $k \times k$ matrix algebra, then $\exp (A)=k^{2}$, while $\exp ^{g r}(A)=2 k^{2}$ (see [10] for details). In the Lie case one can take $L=L_{0} \oplus L_{1}$ to be a two dimensional metabelian algebra with $L_{0}=\langle e\rangle, L_{1}=\langle f\rangle$ and with only one non-trivial product $[e, f]=f$. Then $c_{n}(L)=n-1$ for all $n \geq 2$; hence $\exp (L)=1$. On the other hand, $\exp ^{g r}(L)=2$.

\section{The MAIN CONSTRUCTIONS AND DEFINITIONS}

Let $A$ be an arbitrary non-associative algebra over a field $F$ and let $F\{X\}$ be an absolutely free $F$-algebra with a countable generating set $X$. A polynomial $f=$ $f\left(x_{1}, \ldots, x_{n}\right)$ is said to be an identity of $A$ if $f\left(a_{1}, \ldots, a_{n}\right)=0$ for any $a_{1}, \ldots, a_{n} \in$ $A$. The set of all identities of $L$ forms a T-ideal $\operatorname{Id}(A)$ in $F\{X\}$, that is, an ideal which is stable under all endomorphisms of $F\{X\}$. Denote by $P_{n}=P_{n}\left(x_{1}, \ldots, x_{n}\right)$ the subspace of all multilinear polynomials on $x_{1}, \ldots, x_{n}$ in $F\{X\}$. Then $P_{n} \cap$ $\operatorname{Id}(A)$ is a subspace of all multilinear identities of $A$ of degree $n$. In the case when char $F=0$, the T-ideal $\operatorname{Id}(A)$ is completely determined by the subspaces $\left\{P_{n} \cap \operatorname{Id}(A)\right\}, n=1,2, \ldots$.

For estimating how many identities an algebra $A$ can have, one can define the so-called $n$-th codimension of the identities of $A$ or, for short, the codimension of $A$ :

$$
c_{n}(A)=\operatorname{dim} \frac{P_{n}}{P_{n} \cap \operatorname{Id}(A)}, n=1,2, \ldots .
$$

As was mentioned above, the class of associative and non-associative algebras with exponentially bounded sequence $\left\{c_{n}(A)\right\}$ is sufficiently wide. In the case when $c_{n}(A)<a^{n}$ for some real $a$, one can define the lower and the upper PI-exponents of $A$ as follows:

$$
\underline{\exp }(A)=\liminf _{n \rightarrow \infty} \sqrt[n]{c_{n}(A)}, \quad \overline{\exp }(A)=\limsup _{n \rightarrow \infty} \sqrt[n]{c_{n}(A)}
$$

and the ordinary PI-exponent as follows:

$$
\exp (A)=\lim _{n \rightarrow \infty} \sqrt[n]{c_{n}(A)}
$$

provided that $\exp (A)=\overline{\exp }(A)$.

For $\mathbb{Z}_{2}$-graded algebras one can also consider graded identities. Let $X$ and $Y$ be two infinite sets of variables and let $F\{X \cup Y\}$ be an absolutely free algebra generated by $X \cup Y$. If we suppose that all elements of $X$ are even and all elements of $Y$ are odd, i.e. $\operatorname{deg}(x)=0, \operatorname{deg}(y)=1$ for any $x \in X, y \in Y$, then $F\{X \cup Y\}$ can be 
naturally endowed by a $\mathbb{Z}_{2}$-grading. A polynomial $f=f\left(x_{1}, \ldots, x_{m}, y_{1}, \ldots, y_{n}\right) \in$ $F\{X \cup Y\}$ is said to be a graded identity of a superalgebra $A=A_{0} \oplus A_{1}$ if $f\left(a_{1}, \ldots, a_{m}, b_{1}, \ldots, b_{n}\right)=0$, for all $a_{1}, \ldots, a_{m} \in A_{0}, b_{1}, \ldots, b_{n} \in A_{1}$. Fix $0 \leq k \leq n$ and denote by $P_{k, n-k}$ the subspace of $F\{X \cup Y\}$ spanned by all multilinear polynomials in $x_{1}, \ldots, x_{k} \in X, y_{1}, \ldots, y_{n-k} \in Y$. Then $P_{k, n-k} \cap \operatorname{Id}(A)$ is the set of all multilinear polynomial identities of the superalgebra $A=A_{0} \oplus A_{1}$ in $k$ even and $n-k$ odd variables.

One of the equivalent definitions of graded codimensions of $A$ is

$$
c_{n}^{g r}(A)=\sum_{k=0}^{n}\left(\begin{array}{l}
n \\
k
\end{array}\right) c_{k, n-k}(A),
$$

where

$$
c_{k, n-k}(A)=\operatorname{dim} \frac{P_{k, n-k}}{P_{k, n-k} \cap \operatorname{Id}(A)} .
$$

Starting from a $\mathbb{Z}_{2}$-graded algebra of some class (Lie, Jordan alternative, etc.), one can construct a $\mathbb{Z}_{2}$-graded algebra of a different class using the notion of a Grassmann envelope - they play an exceptional role in PI-theory. For example, any variety of associative algebras is generated by the Grassmann envelope of some finite dimensional associative superalgebra 14. In the Lie case any so-called special variety is generated by the Grassmann envelope of a finitely generated Lie superalgebra 22$]$.

We recall this construction for the Lie and the super Lie cases. Let $G$ be the Grassmann algebra generated by 1 and the infinite set $\left\{e_{1}, e_{2}, \ldots\right\}$ satisfying the following relations: $e_{i} e_{j}=-e_{j} e_{i}, i, j=1,2, \ldots$ It is known that $G$ has a natural $\mathbb{Z}_{2}$-grading $G=G_{0} \oplus G_{1}$, where

$$
\begin{gathered}
G_{0}=\operatorname{Span}\left\langle e_{i_{1}} \cdots e_{i_{n}} \mid n=2 k, k=0,1, \ldots\right\rangle, \\
G_{1}=\operatorname{Span}\left\langle e_{i_{1}} \cdots e_{i_{n}} \mid n=2 k+1, k=0,1, \ldots\right\rangle .
\end{gathered}
$$

Given a Lie algebra $L$ with a $\mathbb{Z}_{2}$-grading $L=L_{0} \oplus L_{1}$, its Grassmann envelope

$$
G(L)=L_{0} \otimes G_{0} \oplus L_{1} \otimes G_{1} \subset L \otimes G
$$

is a Lie superalgebra. Vice versa, if $L=L_{0} \oplus L_{1}$ is a Lie superalgebra, then $G(L)$ is an ordinary Lie algebra with a $\mathbb{Z}_{2}$-grading.

\section{Cocharacters of Grassmann envelopes}

The main tool in studying codimension asymptotics is the representation theory of symmetric groups. We refer the reader to [13] for details. The symmetric group $S_{n}$ acts naturally on multilinear polynomials in $F\{X\}$ as

$$
\sigma f\left(x_{1}, \ldots, x_{n}\right)=f\left(x_{\sigma(1)}, \ldots, x_{\sigma(n)}\right) .
$$

Hence $P_{n}$ is an $F S_{n}$-module and $P_{n} \cap \operatorname{Id}(L)$, and also

$$
P_{n}(L)=\frac{P_{n}}{P_{n} \cap \operatorname{Id}(L)}
$$

are $F S_{n}$-modules. The $S_{n}$-character $\chi\left(P_{n}(L)\right)$ is called the $n$-th cocharacter of $L$ and we shall write

$$
\chi_{n}(L)=\chi\left(P_{n}(L)\right)
$$


Recall that any irreducible $F S_{n}$-module corresponds to a partition $\lambda$ of $n, \lambda \vdash n$, $\lambda=\left(\lambda_{1}, \ldots, \lambda_{k}\right)$, where $\lambda_{1} \geq \cdots \geq \lambda_{k}$ are positive integers and $\lambda_{1}+\cdots+\lambda_{k}=n$. By the Maschke Theorem, any finite dimensional $F S_{n}$-module $M$ decomposes into a direct sum of irreducible components, and hence its character $\chi(M)$ has a decomposition

$$
\chi(M)=\sum_{\lambda \vdash n} m_{\lambda} \chi_{\lambda},
$$

where $m_{\lambda}$ are non-negative integers. In particular, for the algebra $L$ we have

$$
\chi(L)=\sum_{\lambda \vdash n} m_{\lambda} \chi_{\lambda} .
$$

Integers $m_{\lambda}$ in (3) are called multiplicities of $\chi_{\lambda}$ in $\chi_{n}(L)$, and $d_{\lambda}=\operatorname{deg} \chi_{\lambda}=$ $\chi_{\lambda}(1)$ are the dimensions of the corresponding irreducible representations. Therefore

$$
c_{n}(L)=\operatorname{dim} P_{n}(L)=\sum_{\lambda \vdash n} m_{\lambda} d_{\lambda} .
$$

For any partition $\lambda=\left(\lambda_{1}, \ldots, \lambda_{k}\right) \vdash n$ one can construct the Young diagram $D_{\lambda}$ containing $\lambda_{1}$ boxes in the first row, $\lambda_{2}$ boxes in the second row, and so on:

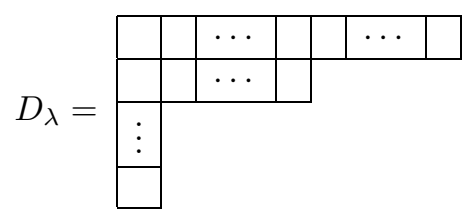

Given integers $k, l, d \geq 0$, we define the partition

$$
h(k, l, d)=(\underbrace{l+d, \ldots, l+d}_{k}, \underbrace{l, \ldots, l}_{d})
$$

of $n=k l+d(k+l)$. The Young diagram associated with $h(k, l, d)$ is hook-shaped, and we define $H(k, l)$, an infinite hook, as the union of all $D_{\lambda}$ with $\lambda=h(k, l, d)$, $d=1,2, \ldots$. For short we shall say that a partition $\lambda \vdash n$ lies in the hook $H(k, l)$, $\lambda \in H(k, l)$, if $D_{\lambda} \subset H(k, l)$. In other words, $\lambda \in H(k, l)$ if $\lambda=\left(\lambda_{1}, \ldots, \lambda_{t}\right)$ and $\lambda_{k+1} \leq l$. According to this definition we shall say that the cocharacter of $L$ lies in the hook $H(k, l)$ if $m_{\lambda}=0$ in (3) as soon as $\lambda \notin H(k, l)$.

A special case of $H(k, l)$ is an infinite strip $H(k, 0)$. In this case $\lambda \in H(k, 0)$ if $\lambda_{k+1}=0$.

The following fact is well-known, and we state it without proof.

Lemma 1. Let $L$ be a finite dimensional algebra, $\operatorname{dim} L=d<\infty$. Then $\chi_{n}(L)$ lies in the hook $H(d, 0)$ for all $n \geq 1$.

Another important numerical invariant of the identities of $L$ is the colength $l_{n}(L)$. By definition

$$
l_{n}(L)=\sum_{\lambda \vdash n} m_{\lambda},
$$

where $m_{\lambda}$ are taken from (3). It easily follows from (4) and (5) that

$$
\max \left\{d_{\lambda} \mid m_{\lambda} \neq 0\right\} \leq c_{n}(L) \leq l_{n}(L) \cdot \max \left\{d_{\lambda} \mid m_{\lambda} \neq 0\right\} .
$$


For studying graded identities of $L=L_{0} \oplus L_{1}$ we need to act separately on even and odd variables. More precisely, the space $P_{k, n-k}=P_{k, n-k}\left(x_{1}, \ldots, x_{k}, y_{1}, \ldots\right.$, $\left.y_{n-k}\right)$ is an $S_{k} \times S_{n-k}$-module where symmetric groups $S_{k}, S_{n-k}$ act on $x_{1}, \ldots, x_{k}$ and $y_{1}, \ldots, y_{n-k}$, respectively. Any irreducible $S_{k} \times S_{n-k}$-module is a tensor product of an $S_{k}$-module and an $S_{n-k}$-module and corresponds to the pair $\lambda, \mu$ of partitions, $\lambda \vdash k, \mu \vdash n-k$. As before, the subspace $P_{n-k} \cap I d(L)$ is an $S_{k} \times S_{n-k}$-stable subspace, and one can consider the quotient

$$
P_{k, n-k}(L)=\frac{P_{k, n-k}}{P_{k, n-k} \cap I d(L)}
$$

as an $S_{k} \times S_{n-k}$-module. Its $S_{k} \times S_{n-k}$-character $\chi_{k, n-k}(L)=\chi\left(P_{k, n-k}(L)\right)$ is decomposed into irreducible components,

$$
\chi_{k, n-k}(L)=\sum_{\substack{\lambda \vdash k \\ \mu \vdash n-k}} m_{\lambda, \mu} \chi_{\lambda, \mu},
$$

and we define the $(k, n-k)$-colength of $L$ as

$$
l_{k, n-k}(L)=\sum_{\substack{\lambda \vdash k \\ \mu \vdash n-k}} m_{\lambda, \mu}
$$

with $m_{\lambda, \mu}$ taken from (7).

First, we prove some relations between graded and non-graded numerical invariants. We begin by recalling the correspondence between multilinear homogeneous polynomials in a free $\mathbb{Z}_{2}$-graded Lie algebra and in a free Lie superalgebra. Let $f=f\left(x_{1}, \ldots, x_{k}, y_{1}, \ldots, y_{m}\right)$ be a non-associative polynomial multilinear on $x_{1}, \ldots, x_{k}, y_{1}, \ldots, y_{m}$, where $x_{1}, \ldots, x_{k}$ are supposed to be even indeterminates and $y_{1}, \ldots, y_{m}$ are supposed to be odd. Then $f$ is a linear combination of monomials from $P_{k, m}$. Let $M=M\left(x_{1}, \ldots, x_{k}, y_{1}, \ldots, y_{m}\right)$ be such a monomial. We fix positions of $y_{1}, \ldots, y_{m}$ in $M$ and write $M$ for short in the following form:

$$
M=X_{0} y_{\sigma(1)} X_{1} \cdots X_{m-1} y_{\sigma(m)} X_{m}
$$

where $X_{0}, \ldots, X_{m}$ are some words (possibly empty) consisting of left and right brackets and indeterminates $x_{1}, \ldots, x_{k}$. Now we define a monomial $\widetilde{M}$ on even indeterminates $x_{1}, \ldots, x_{k}$ and odd indeterminates $y_{1}, \ldots, y_{m}$ from the free Lie superalgebra as

$$
\widetilde{M}=\operatorname{sgn}(\sigma) X_{0} y_{\sigma(1)} X_{1} \cdots X_{m-1} y_{\sigma(m)} X_{m} .
$$

Extending this map $\sim$ by linearity, we obtain a linear isomorphism $P_{k, m} \rightarrow P_{k, m}$ of two subspaces of a $\mathbb{Z}_{2}$-graded free Lie algebra and a free Lie superalgebra, respectively. Although the monomials in $P_{k, m}$ are not linearly independent, it easily follows from the Jacobi and the super-Jacobi identities that the map $\sim$ is welldefined. Similarly, we can define the inverse map from a free Lie superalgebra to a free $\mathbb{Z}_{2}$-graded Lie algebra.

Following the same argument as in the associative case (see [10, Lemma 3.4.7]), we obtain the following result for any $\mathbb{Z}_{2}$-graded Lie algebra $L$ and its Grassmann envelope $G(L)=G_{0} \otimes L_{0} \oplus G_{1} \otimes L_{1}$.

Lemma 2. Let $f \in P_{k, m}$ be a multilinear polynomial in a free Lie algebra L. Then

- $\underset{\sim}{f}$ is a graded identity of $L$ if and only if $\tilde{f}$ is a graded identity of $G(L)$ and - $\widetilde{\widetilde{f}}=f$. 
The next lemma is an obvious generalization of Lemma 1.

Lemma 3. Let $L=L_{0} \oplus L_{1}$ be a finite dimensional Lie algebra, $\operatorname{dim} L_{0}=$ $k, \operatorname{dim} L_{1}=l$, and let

$$
\chi_{q, n-q}(L)=\sum_{\substack{\lambda \vdash q \\ \mu \vdash n-q}} m_{\lambda, \mu} \chi_{\lambda, \mu}
$$

be its $(q, n-q)$-graded cocharacter. If $m_{\lambda, \mu} \neq 0$, then $\lambda \in H(k, 0)$ and $\mu \in H(l, 0)$.

Using this remark we restrict the shape of the graded cocharacter of the Grassmann envelope $G(L)$.

Lemma 4. Let $L=L_{0} \oplus L_{1}$ be a finite dimensional Lie algebra, $\operatorname{dim} L_{0}=$ $k, \operatorname{dim} L_{1}=l$, and let $\widetilde{L}$ be its Grassmann envelope. If

$$
\chi_{q, n-q}(\widetilde{L})=\sum_{\substack{\lambda \vdash q \\ \mu \vdash n-q}} m_{\lambda, \mu} \chi_{\lambda, \mu}
$$

and $m_{\lambda, \mu} \neq 0$ in (요), then $\lambda \in H(k, 0)$ and $\mu \in H(0, l)$.

Proof. Suppose $m_{\lambda, \mu} \neq 0$ in (8) for some $\lambda \vdash q, \mu \vdash n-q$. Then there exists a multilinear polynomial $g=g\left(x_{1}, \ldots, x_{q}, y_{1}, \ldots, y_{n-q}\right)$ such that

$$
f=e_{T_{\lambda}} e_{T_{\mu}} g\left(x_{1}, \ldots, y_{n-q}\right)
$$

is not a graded identity of $\widetilde{L}$, where $e_{T_{\lambda}} \in F S_{q}, e_{T_{\mu}} \in F S_{n-q}$ are essential idempotents generating minimal left ideals in $F S_{q}, F S_{n-q}$, respectively. Inclusion $\lambda \in$ $H(k, 0)$ immediately follows by Lemma 3 since $L$ and $G(L)$ have the same cocharacters on even indeterminates. Since $e_{T_{\lambda}}$ and $e_{T_{\mu}}$ commute, applying Lemma 4.8.6 from [10] we get

$$
\widetilde{f}=a e_{T_{\lambda}} g,
$$

where $a \in I_{\mu^{\prime}}$. Here $\mu^{\prime}$ is the conjugated to $\mu$ partition of $n-q$ and $I_{\mu^{\prime}}$ is the minimal two-sided ideal of $F S_{n-q}$ generated by $e_{T_{\mu^{\prime}}}$. That is, $I_{\mu^{\prime}}$ has the character $r \cdot \chi_{\mu^{\prime}}$, where $r=d_{\mu^{\prime}}=\operatorname{deg} \mu^{\prime}$.

By Lemma $2, \tilde{f}$ is not a graded identity of $G(\widetilde{L})$. Since $\widetilde{\widetilde{h}}=h$ for any $h \in P_{q, n-q}$, we see that $\tilde{f}$ is not a graded identity of $L$ and $\mu^{\prime} \in H(l, 0)$ by Lemma 3. In other words, the number of rows of the Young diagram $D_{\mu^{\prime}}$ does not exceed $l$. This number equals the number of columns of $D_{\mu}$; hence $\mu \in H(0, l)$, and we are done.

Using the previous lemma we restrict the shape of the non-graded cocharacter of $G(L)$.

Lemma 5. Let $L=L_{0} \oplus L_{1}$ be a finite dimensional Lie algebra, $\operatorname{dim} L_{0}=$ $k, \operatorname{dim} L_{1}=l$, and let

$$
\chi(\widetilde{L})=\sum_{\lambda \vdash n} m_{\lambda} \chi_{\lambda}
$$

be the $n$-th (non-graded) cocharacter of $\widetilde{L}=G(L)$. Then $m_{\lambda} \neq 0$ only if $\lambda \in H(k, l)$. 
Proof. Suppose $f \in P_{n}$ is not an identity of $\widetilde{L}$. Since $f$ is multilinear we may assume that $f\left(x_{1}, \ldots, x_{q}, y_{1}, \ldots, y_{n-q}\right) \in P_{q, n-q}$ is not an identity of $\widetilde{L}$ for some $0 \leq q \leq n$. Moreover, we can consider only the case when a graded polynomial $f$ generates in $P_{q, n-q}$ an irreducible $S_{q} \times S_{n-q}$-submodule $M$ with the character $\left(\chi_{\lambda}, \chi_{\mu}\right), \lambda \vdash q, \mu \vdash n-q$.

Now we lift the $S_{q} \times S_{n-q}$-action up to an $S_{n}$-action and consider a decomposition of $F S_{n} M$ into irreducible components:

$$
\chi\left(F S_{n} M\right)=\sum_{\nu \vdash n} m_{\nu} \chi_{\nu} .
$$

Since by Lemma $4 \lambda$ lies in the hook $H(k, 0)$, i.e. the horizontal strip of height $k$, and $\mu$ lies in $H(0, l)$, the vertical strip of width $l$, it follows from the LittlewoodRichardson rule for induced representations ([13, 2.8.13]; see also [10, Thm. 2.3.9]) that $m_{\nu}=0$ as soon as $\nu \notin H(k, l)$, and we have completed the proof.

Lemma 6. Let $G(L)=\widetilde{L}=\widetilde{L}_{0} \oplus \widetilde{L}_{1}$ be the Grassmann envelope of a finite dimensional Lie algebra $L=L_{0} \oplus L_{1}$ with $\operatorname{dim} L_{0}=k, \operatorname{dim} L_{1}=l$. Then its colength sequence $\left\{l_{n}(\widetilde{L})\right\}$ is polynomially bounded.

Proof. We use the notation $\left\{z_{1}, z_{2}, \ldots\right\}$ for non-graded indeterminates here since $\left\{x_{1}, x_{2}, \ldots\right\}$ were even variables in the previous statements.

Let

$$
\chi(\widetilde{L})=\sum_{\lambda \vdash n} m_{\lambda} \chi_{\lambda}
$$

be the $n$-th cocharacter of $\widetilde{L}$. By Lemma 5 we have $\lambda \in H(k, l)$ as soon as $m_{\lambda} \neq 0$ in (9). Fix $\lambda \vdash n$ with $m_{\lambda}=m \neq 0$ and consider the $F S_{n}$-submodule

$$
W_{1} \oplus \cdots \oplus W_{m} \subseteq P_{n}(\widetilde{L})
$$

with $\chi\left(W_{i}\right)=\chi_{\lambda}$, for all $i=1, \ldots, m$.

We shall prove that

$$
m \leq(k+l) 2^{2 k l} n^{k^{2}+l^{2}}
$$

in (10). Denote by $\lambda_{1}^{\prime}, \ldots, \lambda_{l}^{\prime}$ the heights of the first $l$ columns of the Young diagram $D_{\lambda}$. Clearly, it suffices to prove inequality (11) only for $\lambda$ with $\lambda_{k}>l$ and $\lambda_{l}^{\prime}>k$. Otherwise, $\lambda \in H\left(k^{\prime}, l^{\prime}\right)$ with $k^{\prime} \leq k, l^{\prime} \leq l$ and $k^{\prime}+l^{\prime}<k+l$.

Denote

$$
\mu_{1}=\lambda_{1}^{\prime}-k, \ldots, \mu_{l}=\lambda_{l}^{\prime}-k .
$$

Then $\lambda_{1}+\cdots+\lambda_{k}+\mu_{1}+\cdots+\mu_{l}=n$.

It is well-known (see, for example, 29]) that one can choose multilinear $f_{1} \in$ $W_{1}, \ldots, f_{m} \in W_{m}$ such that $F S_{n} f_{1}=W_{1}, \ldots, F S_{n} f_{m}=W_{m}$ and each $f_{i}, i=$ $1, \ldots, m$, is symmetric on $k$ sets of indeterminates of orders $\lambda_{1}, \ldots, \lambda_{k}$ and is alternating on $l$ sets of orders $\mu_{1}, \ldots, \mu_{l}$.

According to this decomposition into symmetric and alternating sets we rename $z_{1}, \ldots, z_{n}$ as follows:

$$
\left\{z_{1}, \ldots, z_{n}\right\}=\left\{z_{1}^{1}, \ldots, z_{\lambda_{1}}^{1}, \ldots, z_{1}^{k}, \ldots, z_{\lambda_{k}}^{k}, \bar{z}_{1}^{1}, \ldots, \bar{z}_{\mu_{1}}^{1}, \ldots, \bar{z}_{1}^{l}, \ldots, \bar{z}_{\mu_{l}}^{l}\right\},
$$

where each $f_{i}$ is symmetric on any set $\left\{z_{1}^{j}, \ldots, z_{\lambda_{j}}^{j}\right\}, j=1, \ldots, k$, and is alternating on any set $\left\{\bar{z}_{1}^{s}, \ldots, \bar{z}_{\mu_{s}}^{s}\right\}, s=1, \ldots, l$. 
We shall find $\delta_{1}, \ldots, \delta_{m} \in F$ such that

$$
f=\delta_{1} f_{1}+\cdots+\delta_{m} f_{m}
$$

is an identity of $\widetilde{L}$ if (11) does not hold. Note that for any $\delta_{1}, \ldots, \delta_{m} \in F$ a polynomial $f$ is also symmetric on each subset $\left\{z_{1}^{i}, \ldots, z_{\lambda_{i}}^{i}\right\}, 1 \leq i \leq k$, and alternating on each subset $\left\{\bar{z}_{1}^{s}, \ldots, \bar{z}_{\mu_{s}}^{s}\right\}, s=1, \ldots, l$.

Let $E=\left\{e_{1}, \ldots, e_{k+l}\right\}$ be a homogeneous basis of $L$ with $E_{0}=\left\{e_{1}, \ldots, e_{k}\right\} \subset L_{0}$, $E_{1}=\left\{e_{k+1}, \ldots, e_{k+l}\right\} \subset L_{1}$. Then $f$ is an identity of $\widetilde{L}$ if and only if $\varphi(f)=0$ for any evaluation $\varphi: Z \rightarrow \widetilde{L}$ such that $\varphi\left(z_{i}\right)=g_{i} \otimes a_{i}, 1 \leq i \leq n$, where $a_{i}$ is a basis element from $E$ and $g_{i} \in G$ has the same parity as $a_{i}$ and $g_{1} \cdots g_{n} \neq 0$ in $G$.

Note also that $\varphi(f)=0$ implies $\varphi^{\prime}(f)=0$ for any evaluation $\varphi^{\prime}$ such that $\varphi^{\prime}\left(z_{i}\right)=g_{i}^{\prime} \otimes a_{i}, 1 \leq i \leq n$, provided that $g_{1} \cdots g_{n} \neq 0$.

Using these two remarks we shall find an upper bound for the number of evaluations for asking the question whether $f$ is an identity of $\widetilde{L}$ or not.

First consider one symmetric subset $Z_{1}=\left\{z_{1}^{1}, \ldots, z_{\lambda_{1}}^{1}\right\}$. If $\varphi\left(z_{i}^{1}\right)=g \otimes e, \varphi\left(z_{j}^{1}\right)=$ $h \otimes e$, for some $i \neq j$ with $e \in E_{1}$, then $\varphi(f)=0$, as follows from the symmetry on $Z_{1}$. Hence we need to check only evaluations with at most $r \leq l$ odd values $\varphi\left(z_{i_{1}}^{1}\right)=g_{1} \otimes e_{t_{1}}, \ldots, \varphi\left(z_{i_{r}}^{1}\right)=g_{r} \otimes e_{t_{r}}$, where $e_{t_{1}}, \ldots, e_{t_{r}} \in E_{1}$ are distinct. Since $Z_{1}$ is the symmetric set of variables, the result of evaluation $\varphi$ does not depend (up to the sign) on the choice of $i_{1}, \ldots, i_{r}$. Hence we have $\left(\begin{array}{l}l \\ r\end{array}\right)$ possibilities.

Given $0 \leq r \leq l$, we estimate the number of evaluations of remaining $\lambda_{1}-r$ variables in the even component of $\widetilde{L}$. First, let $r=0$ and $\varphi\left(z_{i}^{1}\right)=g_{i} \otimes a_{i}, a_{i} \in$ $E_{0}, 1 \leq i \leq \lambda_{1}$. If $e_{1}$ appears in the row $\left(a_{1}, \ldots, a_{\lambda_{1}}\right)$ exactly $\alpha_{1}$ times, $e_{2}$ appears $\alpha_{2}$ times, and so on, then the result of such substitution depends only on $\alpha_{1}, \ldots, \alpha_{k}$ since $f$ is symmetric on $Z_{1}$. Hence we have no more than $\left(\lambda_{1}+1\right)^{k}$ variants since $0 \leq \alpha_{1}, \ldots, \alpha_{k} \leq \lambda_{1}$. In particular, we need at most $(n+1)^{k}$ evaluations if $r=0$.

Now let $r=1$. We can replace by an odd element an arbitrary variable from $Z_{1}$ and get (up to the sign) the same value $\varphi(f)$ since $f$ is symmetric on $Z_{1}$. Suppose, say, that $\varphi\left(z_{\lambda_{1}}^{1}\right)=h \otimes e, e \in E_{1}$, and $\varphi\left(z_{1}^{1}\right)=g_{1} \otimes a_{1}, \ldots, \varphi\left(z_{\lambda_{1}-1}^{1}\right)=g_{\lambda_{1}-1} \otimes a_{\lambda_{1}-1}$, where all $a_{j}$ are even. If $\alpha_{1}, \ldots, \alpha_{k}$ are the same integers as in the case $r=0$, then the result of the substitution also depends only on $\alpha_{1}, \ldots, \alpha_{k}$. Hence for $r=1$ we have at most

$$
\left(\begin{array}{l}
l \\
1
\end{array}\right) \lambda_{1}^{k} \leq\left(\begin{array}{l}
l \\
1
\end{array}\right)(n+1)^{k}
$$

variants for $\varphi$ since $0 \leq \alpha_{1}, \ldots, \alpha_{k} \leq \lambda_{1}-1$.

Similarly, for general $0 \leq r \leq l$ we have at most

$$
\left(\begin{array}{l}
l \\
r
\end{array}\right)\left(\lambda_{1}+1-r\right)^{k} \leq\left(\begin{array}{l}
l \\
r
\end{array}\right)(n+1)^{k}
$$

variants. Therefore, for evaluating all variables from $Z_{1}$ it suffices that

$$
\sum_{r=0}^{l}\left(\begin{array}{l}
l \\
r
\end{array}\right)(n+1)^{k}=2^{l}(n+1)^{k}
$$

substitutions and for all symmetric variables we need at most

$$
\left(2^{l}(n+1)^{k}\right)^{k}
$$

substitutions.

Now consider the alternating set $Z_{1}^{\prime}=\left\{\bar{z}_{1}^{1}, \ldots, \bar{z}_{\mu_{1}}^{1}\right\}$. If $\varphi\left(\bar{z}_{i}^{1}\right)=g \otimes e, \varphi\left(\bar{z}_{j}^{1}\right)=$ $h \otimes e$, for some $i \neq j$ with the same $e \in E_{0}$, then $\varphi(f)=0$. Hence we can choose 
only $0 \leq r \leq k$ distinct basis elements $b_{1}, \ldots, b_{r} \in E_{0}$ for values of $\bar{z}_{i_{1}}^{1}, \ldots, \bar{z}_{i_{r}}^{1}$ of the type $g_{i} \otimes b_{i}$. Up to the sign, the result of the substitution does not depend on $i_{1}, \ldots, i_{r}$, and we have only $\left(\begin{array}{l}k \\ r\end{array}\right)$ options.

Suppose now that all $\varphi\left(\bar{z}_{i}^{1}\right), 1 \leq i \leq r$, are fixed even values. Let

$$
\varphi\left(\bar{z}_{r+1}^{1}\right)=g_{1} \otimes b_{1}, \ldots, \varphi\left(\bar{z}_{\mu_{1}}^{1}\right)=g_{\mu_{1}-r} \otimes b_{\mu_{1}-r}, \quad b_{1} \ldots, b_{\mu_{1}-r} \in E_{1} .
$$

Then (up to the sign) the result of $\varphi$ depends only on the number of entries of $e_{k+1}, \ldots, e_{k+l}$ into the row $\left(b_{1}, \ldots, b_{\mu_{1}-r}\right)$. Hence we have at most $\left(\mu_{1}-r+1\right)^{l}$ variants for the substitution of odd variables. As in the symmetric case we have the following upper bound:

$$
\sum_{r=0}^{k}\left(\begin{array}{l}
k \\
r
\end{array}\right)(n+1)^{l}=2^{k}(n+1)^{l}
$$

for one subset and $\left(2^{k}(n+1)^{l}\right)^{l}$ for all skew variables.

We have proved that one can find $T \leq 2^{k l}(n+1)^{l^{2}+k^{2}}$ evaluations $\varphi_{1}, \ldots, \varphi_{T}$ such that the relations

$$
\varphi_{1}(f)=\cdots=\varphi_{T}(f)=0
$$

imply $\varphi(f)=0$ for any evaluation $\varphi$; that is, $f$ is an identity of $\widetilde{L}$. Recall that $f=\delta_{1} f_{1}+\cdots+\delta_{m} f_{m}$. Therefore for any evaluation $\varphi$ the equality $\varphi(f)=0$ can be viewed as a system of $k+l$ homogeneous linear equations in the algebra $\widetilde{L}$ on unknown coefficients $\delta_{1}, \ldots, \delta_{m}$. If (11) does not hold, then the system (13) has a non-trivial solution $\bar{\delta}_{1}, \ldots, \bar{\delta}_{m}$, and $f=\bar{\delta}_{1} f_{1}+\cdots+\bar{\delta}_{m} f_{m}$ is an identity of $\widetilde{L}$, a contradiction.

We have proved the inequality (11). From this inequality it follows that all multiplicities in (9) are bounded by $(k+l) 2^{2 k l} n^{k^{2}+l^{2}}$. Finally, note that the number of partitions $\lambda \in H(k, l)$ is bounded by $n^{k+l}$. Hence

$$
l_{n}(\widetilde{L})<(k+l) 2^{2 k l} n^{k^{2}+l^{2}+k l},
$$

and we have thus completed the proof.

As a corollary of previous results we obtain the following:

Proposition 1. Let $L=L_{0} \oplus L_{1}$ be a finite dimensional $\mathbb{Z}_{2}$-graded Lie algebra with $\operatorname{dim} L_{0}=k, \operatorname{dim} L_{1}=l$ and let $\widetilde{L}=G(L)$ be its Grassmann envelope. Then there exist constants $\alpha, \beta \in \mathbb{R}$ such that

$$
c_{n}(\widetilde{L}) \leq \alpha n^{\beta}(k+l)^{n} .
$$

In particular,

$$
\overline{\exp }(\widetilde{L})=\limsup _{n \rightarrow \infty} \sqrt[n]{c_{n}(\widetilde{L})} \leq k+l .
$$

Proof. By [10, Lemma 6.2.5], there exist constants $C$ and $r$ such that

$$
\sum_{\lambda \in H(k, l)} d_{\lambda} \leq C n^{r}(k+l)^{n}
$$

for all $n=1,2, \ldots$. In particular,

$$
\max \left\{d_{\lambda} \mid \lambda \vdash n, \lambda \in H(k, l)\right\} \leq C n^{r}(k+l)^{n} .
$$

Now Lemma 6] and the inequality (6) complete the proof. 


\section{Existence of PI-EXPonents}

Proposition 2. Let $L$ be a finite dimensional simple Lie algebra over an algebraically closed field of characteristic zero with some $\mathbb{Z}_{2}$-grading, $L=L_{0} \oplus L_{1}$, $\operatorname{dim} L_{0}=k, \operatorname{dim} L_{1}=l$. Also let $\widetilde{L}=G(L)$ be its Grassmann envelope. Then there exist constants $\gamma>0, \delta \in \mathbb{R}$ such that

$$
c_{n}(\widetilde{L}) \geq \gamma n^{\delta}(k+l)^{n} .
$$

In particular,

$$
\underline{\exp }(\widetilde{L})=\liminf _{n \rightarrow \infty} \sqrt[n]{c_{n}(\widetilde{L})} \geq k+l .
$$

Proof. Denote $d=k+l=\operatorname{dim} L$. By [19, Theorem 12.1], for the adjoint representation of $L$ there exists a multilinear asssociative polynomial $h=h\left(u_{1}^{1}, \ldots, u_{d}^{1}, \ldots\right.$, $\left.u_{1}^{m}, \ldots, u_{d}^{m}\right)$ alternating on each subset of indeterminates $\left\{u_{1}^{i}, \ldots, u_{d}^{i}\right\}, 1 \leq i \leq m$, such that under any evaluation $\varphi: u_{j}^{i} \rightarrow a d b_{j}^{i}, b_{j}^{i} \in L$, the value $\varphi(h)$ is a scalar linear transformation of $L$ and $\varphi(h) \neq 0$ for some $h$. It follows that for any integer $t \geq 1$ there exists a multilinear Lie polynomial

$$
f_{t}=f_{t}\left(u_{1}^{1}, \ldots, u_{d}^{1}, \ldots, u_{1}^{m t}, \ldots, u_{d}^{m t}, w\right)
$$

alternating on each set $\left\{u_{1}^{i}, \ldots, u_{d}^{i}\right\}, 1 \leq i \leq m t$, such that $\varphi\left(f_{t}\right) \neq 0$ for some evaluation $\varphi:\left\{u_{1}^{1}, \ldots, u_{d}^{m t}, w\right\} \rightarrow L_{0} \cup L_{1}$. Since $f_{t}$ is multilinear and alternating on each set $\left\{u_{1}^{i}, \ldots, u_{d}^{i}\right\}$ and $d=\operatorname{dim} L_{0}+\operatorname{dim} L_{1}$, it follows that for any $t \geq 1$ we get a graded multilinear polynomial

$$
f_{t}=f_{t}\left(x_{1}^{1}, \ldots, x_{k}^{1}, \ldots, x_{1}^{m t}, \ldots, x_{k}^{m t}, y_{1}^{1}, \ldots, y_{l}^{1}, \ldots, y_{1}^{m t}, \ldots, y_{l}^{m t}, w\right)
$$

which is not a graded identity of $L$ and is alternating on each subset $\left\{x_{1}^{i}, \ldots, x_{k}^{i}\right\}$ and on each subset $\left\{y_{1}^{i}, \ldots, y_{l}^{i}\right\}, 1 \leq i \leq m t$, where $x_{j}^{i}$ 's are even and $y_{j}^{i}$ 's are odd variables. The latter indeterminate $w$ can be taken of arbitrary parity; say, $w=x_{0}$ is even.

Consider an $S_{p} \times S_{q}$-action on

$$
P_{p+1, q}=P_{p+1, q}\left(x_{0}, x_{1}^{1}, \ldots, x_{k}^{m t}, y_{1}^{1}, \ldots, y_{l}^{m t}\right),
$$

where $p=m t k, q=m t l$ and $S_{p}, S_{q}$ act on $\left\{x_{j}^{i}\right\},\left\{y_{j}^{i}\right\}$, respectively. It follows from Lemma 3 that the $S_{p} \times S_{q}$-character of the submodule generated by $f$ in $P_{p+1, q}$ lies in the pair of strips $H(k, 0), H(l, 0)$, that is,

$$
\chi\left(F\left[S_{p} \times F_{q}\right] f\right)=\sum_{\substack{\lambda \vdash p \\ \mu \vdash q}} m_{\lambda, \mu} \chi_{\lambda, \mu}
$$

with $m_{\lambda, \mu}=0$, unless $\lambda \in H(k, 0), \mu \in H(l, 0)$. Hence $\lambda$ is a partition of $m t k$ with at most $k$ rows. On the other hand, $f$ depends on $m t$ alternating subsets of even indeterminates of order $k$ each. It is well-known that in this case $m_{\lambda, \mu}=0$ if $\lambda=\left(\lambda_{1}, \lambda_{2}, \ldots\right)$ and $\lambda_{1} \geq m t+1$. It follows that only the rectangular partition

$$
\lambda=(\underbrace{m t, \ldots, m t}_{k})
$$

can appear in $F\left[S_{p} \times F_{q}\right] f$ with non-zero multiplicity. Similarly,

$$
\mu=(\underbrace{m t, \ldots, m t}_{l})
$$


if $m_{\lambda, \mu} \neq 0$. Hence we can assume that $f$ has the form

$$
f=e_{T_{\lambda}} e_{T_{\mu}} g\left(x_{1}^{1}, \ldots, y_{l}^{m t}, w\right)
$$

with $\lambda$ and $\mu$ of the types (14), (15), respectively.

By Lemma 2, the polynomial $\tilde{f}$ is not an identity of the Lie superalgebra $\widetilde{L}=G(L)$, and by Lemma 4.8.6 from [10, the graded polynomial $\tilde{f}$ generates in $P_{p+1, q}(\widetilde{L})$ an irreducible $S_{p} \times S_{q^{-}}$-submodule with the character $\left(\chi_{\lambda}, \chi_{\mu^{\prime}}\right)$, where

$$
\mu^{\prime}=(\underbrace{l, \ldots, l}_{m t})
$$

is conjugated to a $\mu$ partition of $m t l$.

First we apply the Littlewood-Richardson rule and induce this $S_{p} \times S_{q}$-module up to an $S_{n}$-module. Then we induce the obtained $S_{n}$-module up to an $S_{n+1}$-module, where $n=p+q=m t(k+l)$. It follows from the Littlewood-Richardson rule that the induced $S_{n+1}$-module can contain only a simple submodule corresponding to partitions $\nu \vdash n+1$ such that the Young diagram $D_{\nu}$ contains a subdiagram $D_{\nu_{0}}$, where

$$
\nu_{0}=h\left(k, l, t_{0}\right)=(\underbrace{l+t_{0}, \ldots, l+t_{0}}_{k}, \underbrace{l, \ldots, l}_{t_{0}})
$$

is a finite hook with $t_{0} \geq l-k, m t-k l$. Since we are interested in an asymptotic of codimensions, we may assume that $m t-k l>l-k$ and then $t_{0}=m t-k l$. In particular, $\nu_{0}$ is a partition of $n_{0}=(k+l) t_{0}+k l$. Then $n+1-n_{0}=(k+l-1) k l+1$, and by [10, Lemma 6.2.4]

$$
d_{\nu_{0}} \leq d_{\nu} \leq n^{c} d_{\nu_{0}}
$$

where $c=(k+l-1) k l+1$ and

$$
d_{h\left(k, l, t_{0}\right)} \simeq a n_{0}^{b}(k+l)^{n_{0}} \quad \text { if } n_{0} \rightarrow \infty
$$

for some constants $a, b$ by Lemma 6.2.5 from [10. Here the relation $f(n) \simeq g(n)$ means that $\lim _{n \rightarrow \infty} \frac{f(n)}{g(n)}=1$. Since $c_{n+1}(\widetilde{L}) \geq d_{\nu}$ we get the inequality

$$
c_{n+1}(\widetilde{L}) \geq \alpha(n+1)^{\beta}(k+l)^{n+1}
$$

for all $n=m(k+l) t, t=1,2, \ldots$, for some constants $\alpha>0$ and $\beta$.

Since the Lie algebra $L$ is simple, the Grassmann envelope $\widetilde{L}$ is a centerless Lie superalgebra. It is not difficult to see that in this case $c_{r+1}(\widetilde{L}) \geq c_{r}(\widetilde{L})$, for all $r \geq 1$. Hence by (16) we have

$$
c_{n+j}(\widetilde{L}) \geq \alpha(n+1)^{\beta}(k+l)^{n+1}
$$

for any $1 \leq j \leq m(k+l)$. Since $n=m(k+l) t$ one can find constants $\gamma>0$ and $\delta$ such that

$$
c_{r}(\widetilde{L}) \geq \gamma r^{\delta}(k+l)^{r}
$$

for all positive integers $r$, and we have completed the proof.

Theorem 11 now easily follows from Propositions 1 and 2 
Proof of Theorem 2. First we obtain an upper bound for $c_{n}^{g r}(\widetilde{L})$ :

$$
c_{n}^{g r}(\widetilde{L})=\sum_{q=0}^{n}\left(\begin{array}{l}
n \\
q
\end{array}\right) c_{q, n-q}(\widetilde{L}),
$$

where

$$
c_{q, n-q}(\widetilde{L})=\sum_{\substack{\lambda \vdash q \\ \mu \vdash n-q}} m_{\lambda, \mu} d_{\lambda, \mu}
$$

and $d_{\lambda, \mu}=\operatorname{deg} \chi_{\lambda, \mu}=\operatorname{deg} \chi_{\lambda} \cdot \operatorname{deg} \chi_{\mu}=d_{\lambda} d_{\mu}$. Moreover, $\lambda \in H(k, 0), \mu \in H(0, l)$ by Lemma 4. Applying Lemma 6.2.5 from [10, we obtain

$$
\sum_{\substack{\lambda \in H(k, 0) \\ \lambda \vdash q}} d_{\lambda} \leq C n^{r} k^{q}, \quad \sum_{\substack{\mu \in H(0, l) \\ \mu \vdash n-q}} d_{\mu} \leq C n^{r} l^{n-q}
$$

for some constants $C, r$, and hence

$$
\sum_{\substack{\lambda \in H(k, 0), \lambda \vdash q \\ \mu \in H(0, l), \mu \vdash n-q}} d_{\lambda} d_{\mu} \leq C^{2} n^{2 r} k^{q} l^{n-q} .
$$

On the other hand, the graded colength

$$
l_{q, n-q}(\widetilde{L})=\sum_{\substack{\lambda \vdash q \\ \mu \vdash n-q}} m_{\lambda, \mu}
$$

is not greater than the non-graded colength $l_{n}(\widetilde{L})$. Since $l_{n}(\widetilde{L})$ is polynomially bounded by Lemma 6. one can find a polynomial $\varphi(n)$ such that

$$
m_{\lambda, \mu} \leq \varphi(n)
$$

for any $m_{\lambda, \mu}$ in (17). It now follows from (17), (18) and (19) that for $\psi(n)=$ $C^{2} n^{2 r} \varphi(n)$ we have

$$
c_{n}^{g r}(\widetilde{L}) \leq \psi(n) \sum_{q=1}^{n}\left(\begin{array}{l}
n \\
q
\end{array}\right) k^{q} l^{n-q}=\psi(n)(k+l)^{n},
$$

and we have obtained an upper bound for $c_{n}^{g r}((\widetilde{L}))$.

On the other hand, it was proved in [2, Lemma 3.1] that for any associative $G$-graded algebra $A$, where $G$ is a finite group, an ordinary $n$-th codimension is less than or equal to the graded $n$-th codimension, for any $n$. Proof of this lemma does not use associativity. Hence

$$
c_{n}^{g r}(\widetilde{L}) \geq c_{n}(\widetilde{L}),
$$

and Theorem 2 now follows from (20), (21) and Proposition 2 ,

\section{REFERENCES}

[1] Yuri Bahturin and Vesselin Drensky, Graded polynomial identities of matrices, Linear Algebra Appl. 357 (2002), 15-34, DOI 10.1016/S0024-3795(02)00356-7. MR.1935223 (2003k:16034)

[2] Yuri Bahturin, Antonio Giambruno, and David M. Riley, Group-graded algebras with polynomial identity, Israel J. Math. 104 (1998), 145-155, DOI 10.1007/BF02897062. MR.1622291 (99c:16041)

[3] A. Berele and A. Regev, Applications of hook Young diagrams to P.I. algebras, J. Algebra 82 (1983), no. 2, 559-567, DOI 10.1016/0021-8693(83)90167-9. MR704771 (84g:16012) 
[4] Allan Berele and Amitai Regev, On the codimensions of the verbally prime P.I. algebras, Israel J. Math. 91 (1995), no. 1-3, 239-247, DOI 10.1007/BF02761648. MR.1348314 (96g:16028)

[5] A. Giambruno, S. Mishchenko, and M. Zaicev, Codimensions of algebras and growth functions, Adv. Math. 217 (2008), no. 3, 1027-1052, DOI 10.1016/j.aim.2007.07.008. MR.2383893 (2008m:17005)

[6] Antonio Giambruno, Amitai Regev, and Michail Zaicev, On the codimension growth of finitedimensional Lie algebras, J. Algebra 220 (1999), no. 2, 466-474, DOI 10.1006/jabr.1999.7933. MR:1717352 (2001h:17011)

[7] Antonio Giambruno, Amitai Regev, and Michail V. Zaicev, Simple and semisimple Lie algebras and codimension growth, Trans. Amer. Math. Soc. 352 (2000), no. 4, 1935-1946, DOI 10.1090/S0002-9947-99-02419-8. MR.1637070(2000i:17006)

[8] A. Giambruno and M. Zaicev, On codimension growth of finitely generated associative algebras, Adv. Math. 140 (1998), no. 2, 145-155, DOI 10.1006/aima.1998.1766. MR1658530 (99k:16049)

[9] A. Giambruno and M. Zaicev, Exponential codimension growth of PI algebras: an exact estimate, Adv. Math. 142 (1999), no. 2, 221-243, DOI 10.1006/aima.1998.1790. MR.1680198 (2000a:16048)

[10] Antonio Giambruno and Mikhail Zaicev, Polynomial identities and asymptotic methods, Mathematical Surveys and Monographs, vol. 122, American Mathematical Society, Providence, RI, 2005. MR2176105 (2006g:16054)

[11] Antonio Giambruno and Mikhail Zaicev, Codimension growth of special simple Jordan algebras, Trans. Amer. Math. Soc. 362 (2010), no. 6, 3107-3123, DOI 10.1090/S0002-9947-0904865-X. MR 2592948(2011e:17048)

[12] Antonio Giambruno and Mikhail Zaicev, On codimension growth of finite-dimensional Lie superalgebras, J. Lond. Math. Soc. (2) 85 (2012), no. 2, 534-548, DOI 10.1112/jlms/jdr059. MR2901077

[13] Gordon James and Adalbert Kerber, The representation theory of the symmetric group, Encyclopedia of Mathematics and its Applications, vol. 16, Addison-Wesley Publishing Co., Reading, Mass., 1981. With a foreword by P. M. Cohn; With an introduction by Gilbert de B. Robinson. MR644144 (83k:20003)

[14] Aleksandr Robertovich Kemer, Ideals of identities of associative algebras, Translations of Mathematical Monographs, vol. 87, American Mathematical Society, Providence, RI, 1991. Translated from the Russian by C. W. Kohls. MR1108620 (92f:16031)

[15] S. P. Mishchenko, On the problem of the Engel property, Mat. Sb. (N.S.) 124(166) (1984), no. 1, 56-67 (Russian). MR743057(86i:17005)

[16] S. P. Mishchenko, Growth of varieties of Lie algebras, Uspekhi Mat. Nauk 45 (1990), no. 6(276), 25-45, 189, DOI 10.1070/RM1990v045n06ABEH002710 (Russian); English transl., Russian Math. Surveys 45 (1990), no. 6, 27-52. MR1101331(92g:17003)

[17] S. P. Mishchenko and V. M. Petrogradsky, Exponents of varieties of Lie algebras with a nilpotent commutator subalgebra, Comm. Algebra 27 (1999), no. 5, 2223-2230, DOI 10.1080/00927879908826560. MR1683862(2000d:17010)

[18] V. M. Petrogradsky, Scale for codimension growth of Lie algebras, Methods in ring theory (Levico Terme, 1997), Lecture Notes in Pure and Appl. Math., vol. 198, Dekker, New York, 1998, pp. 213-222. MR 1767980

[19] Yu. P. Razmyslov, Identities of algebras and their representations, Translations of Mathematical Monographs, vol. 138, American Mathematical Society, Providence, RI, 1994. Translated from the 1989 Russian original by A. M. Shtern. MR1291603 (95i:16022)

[20] Amitai Regev, Existence of identities in $A \otimes B$, Israel J. Math. 11 (1972), 131-152. MR0314893(47 \#3442)

[21] Amitai Regev, Codimensions and trace codimensions of matrices are asymptotically equal, Israel J. Math. 47 (1984), no. 2-3, 246-250, DOI 10.1007/BF02760520. MR.738172 (85j:16024)

[22] A. Ya. Vaŭs, Special varieties of Lie algebras, Algebra i Logika 28 (1989), no. 1, 29-40, 122, DOI 10.1007/BF01980605 (Russian); English transl., Algebra and Logic 28 (1989), no. 1, 17-24. MR1061855 (91e:17025)

[23] M. V. Zaĭtsev, Identities of affine Kac-Moody algebras, Vestnik Moskov. Univ. Ser. I Mat. Mekh. 2 (1996), 33-36, 104 (Russian, with Russian summary); English transl., Moscow Univ. Math. Bull. 51 (1996), no. 2, 29-31. MR1486810 
[24] M. V. Zaĭtsev, Varieties of affine Kac-Moody algebras, Mat. Zametki 62 (1997), no. 1, 95-102, DOI 10.1007/BF02356067 (Russian, with Russian summary); English transl., Math. Notes 62 (1997), no. 1-2, 80-86 (1998). MR1619988 (99g:17013)

[25] M. V. Zaĭtsev, Integrality of exponents of growth of identities of finite-dimensional Lie algebras, Izv. Ross. Akad. Nauk Ser. Mat. 66 (2002), no. 3, 23-48, DOI 10.1070/IM2002v066n03ABEH000386 (Russian, with Russian summary); English transl., Izv. Math. 66 (2002), no. 3, 463-487. MR.1921808 (2003g:17004)

[26] M. V. Zaĭtsev and S. P. Mishchenko, Varieties of Lie superalgebras of polynomial growth, Uspekhi Mat. Nauk 52 (1997), no. 2(314), 165-166, DOI 10.1070/RM1997v052n02ABEH001804 (Russian); English transl., Russian Math. Surveys 52 (1997), no. 2, 432-433. MR1480152

[27] M. V. Zaĭtsev and S. P. Mishchenko, A criterion for the polynomial growth of varieties of Lie superalgebras, Izv. Ross. Akad. Nauk Ser. Mat. 62 (1998), no. 5, 103-116, DOI 10.1070/im1998v062n05ABEH000217 (Russian, with Russian summary); English transl., Izv. Math. 62 (1998), no. 5, 953-967. MR.1680888 (2000g:17004)

[28] M. V. Zaicev and S. P. Mishchenko, An example of a variety of Lie algebras with a fractional exponent, J. Math. Sci. (New York) 93 (1999), no. 6, 977-982, DOI 10.1007/BF02366352. Algebra, 11. MR1698766 (2000f:17016)

[29] M. V. Zaĭtsev and S. P. Mishchenko, On the colength of varieties of linear algebras, Mat. Zametki 79 (2006), no. 4, 553-559, DOI 10.1007/s11006-006-0056-0 (Russian, with Russian summary); English transl., Math. Notes 79 (2006), no. 3-4, 511-517. MR2251143 (2007d:17006)

[30] M. V. Zaitsev and S. P. Mishchenko, The growth of some varieties of Lie superalgebras, Izv. Ross. Akad. Nauk Ser. Mat. 71 (2007), no. 4, 3-18, DOI 10.1070/IM2007v071n04ABEH002371 (Russian, with Russian summary); English transl., Izv. Math. 71 (2007), no. 4, 657-672. MR2360005 (2008h:17007)

[31] M. V. Zaĭtsev and S. P. Mishchenko, Identities of Lie superalgebras with a nilpotent commutator, Algebra Logika 47 (2008), no. 5, 617-645, 649, DOI 10.1007/s10469-008-9022-0 (Russian, with Russian summary); English transl., Algebra Logic 47 (2008), no. 5, 348-364. MR2508321 (2010h:17005)

Faculty of Education and Faculty of Mathematics and Physics, University of LuUbljana, Kardeljeva Pl. 16, Ljubljana, 1000 Slovenia

E-mail address: dusan.repovs@guest.arnes.si

Department of Algebra, Faculty of Mathematics and Mechanics, Moscow State University, Moscow, 119992 Russia

E-mail address: zaicevmv@mail.ru 